\title{
Transcriptomic Time Series Analysis in Wound Healing: Challenges and Perspectives on Data Interpretation
}

\section{Ksenia Zlobina ( $\nabla$ kzlobina@ucsc.edu )}

University of California, Santa Cruz

\section{Eric Malekos}

University of California, Santa Cruz

Han Chen

University of California, Santa Cruz

Marcella Gomez

University of California, Santa Cruz

\section{Research Article}

Keywords: Transcriptomic time series analysis, wound healing, data interpretation

Posted Date: October 6th, 2021

DOI: https://doi.org/10.21203/rs.3.rs-929173/v1

License: (c) (i) This work is licensed under a Creative Commons Attribution 4.0 International License.

Read Full License 


\section{challenges and perspectives on data interpretation}

3

4

8 Abstract

9 Background: Wound transcriptomic analysis can be used to quantify wound healing stages and peaks of expression for highly differentially expressed genes are lost. Nevertheless, we derived a 21 short list of genes highly differentially expressed in all datasets under consideration. After
K. Zlobina*, E. Malekos, H. Chen, M. Gomez

University of California, Santa Cruz

*Corresponding author identify leverage points for wound healing intervention. However, individual gene signatures corresponding to wound healing stages vary from one experiment to another and are highly dependent on both experimental setup and bioinformatics methods.

Methods: We develop a systematic approach to informatively compare time series from publicly available wound transcriptomic datasets, including mouse and human wounds, and identify consistent gene expression patterns.

Results: We reveal the limitations of gene expression data collection, interpretation, and comparison. For example, the sample rate of wound transcriptomic sample collection must be higher than the rate of changes in the wound healing processes, otherwise, important changes in gene expression may be missed. This may lead to mis finding the most significant genes, as 
22 clustering and normalization, these genes clearly demonstrate similarly changing dynamics of

23 expression between the wounds and may be used for wound healing stage detection.

24 Conclusions: A list of genes that may be used for transcriptomics-based wound healing stage

25 detection is provided. In addition, we suggest experimental approaches that could help

26 researchers to extract more meaningful results.

\section{Introduction}

Wound healing is a dynamic process involving a series of coordinated biological processes

30 partitioned by scientist into four distinct stages [Canedo-Dorantes 2019]: hemostasis,

31 inflammation [Kim, 2008; Krzyszczyk, 2018], proliferation and remodeling [Bainbridge, 2013;

32 Pastar, 2014]. Many cell types participate in wound healing [Wilkinson 2020], giving rise to

33 expression of different genes [Brant 2015; Deonarine 2007].

34 Transcriptome microarrays detect expression of tens of thousands of genes. [Blumenberg

35 2019; Tachibana 2015]. Transcriptomic analysis of cutaneous wound biopsies is particularly

36 promising because the rapidly changing wound environment produces many activation stimuli

37 that induce cellular activation reactions that are not fully understood [Deonarine 2007; Chen

38 2010]. Until now, only a few experiments with skin wound transcriptomic analysis have been

39 done [GSE460, GSE8056, Greco 2010, GSE23006, Chen 2010].

40 Despite differences in wound experimental conditions, wound healing stages are

41 assumed to be the same. Thus, common properties of gene expression should exist across

42 wounds. Comparison of transcriptomic data from different wounds was made previously as a 
43 meta-analysis of many datasets from different tissue wounds [Sass, 2018]. The researchers

44 identified several groups of genes that changed expression in most wounds, but not in all.

45 In this manuscript, we look for genes showing universal expression patterns in different

46 wounds that could serve as wound stage indicators. We restrict ourselves to comparisons

47 between skin wounds. Corresponding datasets are [GSE460, GSE8056, GSE23006] - human and

48 mice burn wounds and mice surgical wounds. For translational work, we present a method to

49 identify patterns in gene expression that are consistent across varying wound experimental

50 conditions and species. This work assess the potential of transcriptomes for data-based

51 predictive models.

52 To fairly compare across studies, a systematic and consistent approach to identifying

53 differentially expressed genes is applied to all datasets. We identify orthologous genes between

54 mouse and human, only genes with orthologs in all three datasets are considered. We come out

55 with a short list of 58 genes highly differentially expressed in all 3 datasets under consideration.

56 After clustering and normalization, these genes clearly demonstrate similarly changing dynamics

57 of expression between the wounds.

$58 \quad$ Additionally, we make note of methodological problems in wound transcriptomic analysis

59 and emphasize which shortcomings should be resolved in future experiments to improve the

60 power of this experimental tool for wound investigation.

61

\section{Data preparation}


63 Consider 3 transcriptomic datasets from wounds: GSE8056 contains human skin burn wound.

64 GSE460 contains mouse skin burn wound. GSE23006 [Chen 2010] is mouse surgical 1mm wound

65 dataset containing both skin and tongue, of which we consider only the skin data.

66

67 To arrive at a list of genetic biomarkers associated with each wound healing stage we search for

68 genes that are reliably highly differentially expressed in all wounds. The following requirements

69 are imposed in our data pre-processing:

70 - Experimental error must be minimized. That is, we rely on genes with low biological

71 dispersion.

72 - A link between mouse and human genes must be found.

73 - To make a direct comparison, each gene must be presented only once in each dataset.

74 All replicates and repeated measurements must be analyzed, and one replicate or mean

$75 \quad$ value accepted.

76 - Only genes that are present in all datasets may be compared.

77

78 To compare gene expression between wounds, we apply several filtering procedures described

79 in "Methods". The number of genes left in each dataset at each filtering step is presented in Table

801 . We emphasize that while we tried to come up with a standardized approach, other approaches

81 can be considered.

82

83 Table 1. Numbers of genes in each dataset after each filtering step (described in Methods).

\begin{tabular}{|l|l|l|l|}
\hline Dataset & GSE23006 & GSE460 & GSE8056 \\
\hline
\end{tabular}




\begin{tabular}{|l|l|l|l|}
\hline & mouse & mouse & human \\
& skin & burn skin & skin \\
wound & wound & wound \\
\hline Initial number of genes & 45101 & 7275 & 54675 \\
\hline After filtering multiple genes in same row (1) & 29309 & 6130 & 31762 \\
\hline After filtering based on consistency of replicates (2) & 26519 & 5760 & 30242 \\
\hline Unique gene names in filtered subset & 13626 & 4980 & 13777 \\
\hline After filtering of repeated measurements of same & 8005 & 4449 & 9254 \\
gene in each dataset (3) & & & \\
\hline After filtering several genes corresponding to the & 7937 & 4441 & 9249 \\
\hline same homologene number (4) & & & \\
\hline
\end{tabular}

Intersections of filtered datasets

87 The 3 datasets contain 7937 (GSE23006), 4441 (GSE460) and 9249 (GSE8056) genes after filtering.

88 The intersections contain even less genes, see Table 2.

89

90 Table 2. Number of genes in the intersections of each pair of datasets after filtration

\begin{tabular}{|l|l|l|}
\hline Dataset 1 & Dataset 2 & N of common genes \\
\hline GSE23006 mouse surg (7937) & GSE460 mouse burn (4441) & 2441 \\
\hline GSE23006 mouse surg (7937) & GSE8056 human (9249) & 5278 \\
\hline GSE460 mouse burn (4441) & GSE8056 human (9249) & 2855 \\
\hline
\end{tabular}


91 Intersection of all 3 datasets consists of 1622 genes.

92

\section{Data characterization and comparison}

\section{Fold change as indicator of highly differentially expressed genes}

95 To find the most significant genes, researchers often assign some rank to each gene and select a

96 final gene list based on it. Though not universal [Dalman 2012; McCarthy 2009], in many papers

97 the maximal fold change is used as an indicator. Suppose that gene expression during wound

98 healing is given by a vector of intensities: $\vec{g}=\left[g\left(t_{0}\right), g\left(t_{1}\right), \cdots, g\left(t_{k}\right)\right]$ at time points $t_{0}, t_{1}, \ldots, t_{k}$.

99 Then the maximal up- or down-regulation fold change may be found by formulas

$100 \Delta G_{u}=\frac{I_{\max }}{I_{0}}$ and $\Delta G_{d}=\frac{I_{\min }}{I_{0}}$, respectively,

101 where $I_{\max }=\max \left(g\left(t_{i}\right)\right), I_{\min }=\min \left(g\left(t_{i}\right)\right)$, and $I_{0}=g\left(t_{0}\right)$. Here, we introduce the 102 relative maximum observed fold change

103

$$
\Delta G=\frac{I_{\max }-I_{\min }}{I_{\min }}
$$

104 To account for variations in baseline expressions across datasets. To find common and unique 105 features of the wounds considered, we try to differentiate genes that are commonly differentially 106 expressed in all wounds from those with high variation across wounds. In other words, we ask 107 how many genes have approximately the same value of fold change in all wounds.

Figure 1 shows the distribution of genes by the value of fold change $\Delta G$. As seen from

109 Figure 1, there is no "natural" threshold value for $\Delta G$ to distinguish between differentially 110 expressed genes and non-differentially expressed. The amount of differentially expressed genes 111 fully depends on the researcher's choice of the threshold for $\Delta G$. 


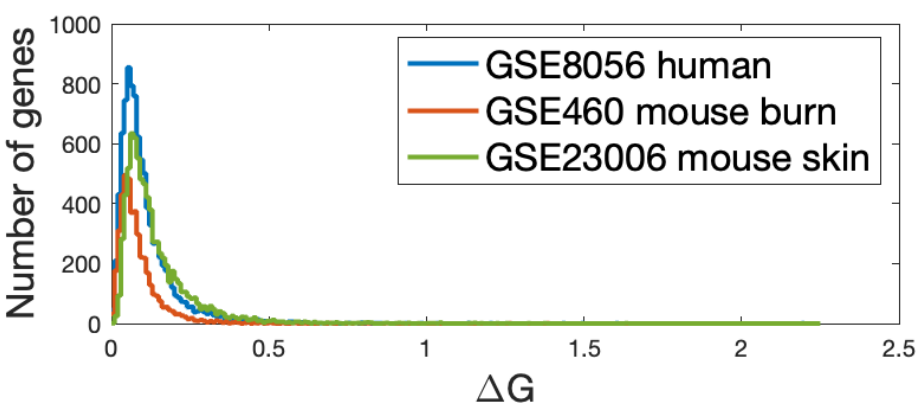

Figure 1 Distribution of genes by the value of fold change $\Delta G$.

Figure 2 demonstrates the relation of $\Delta G$ for each gene between datasets. Each gene is 116 presented by one point with abscissa $\Delta G$ from one dataset and ordinate $\Delta G$ from another

117 dataset. One can see that there is no strong correlation of $\Delta G$ between each pair of wounds with 118 Spearman rank correlation coefficients calculated in Table 1.

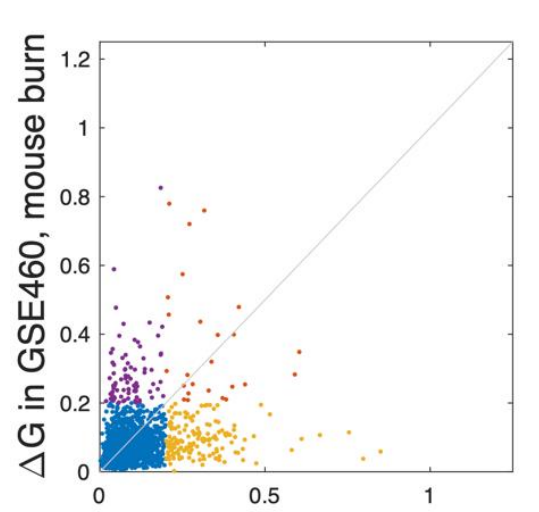

120

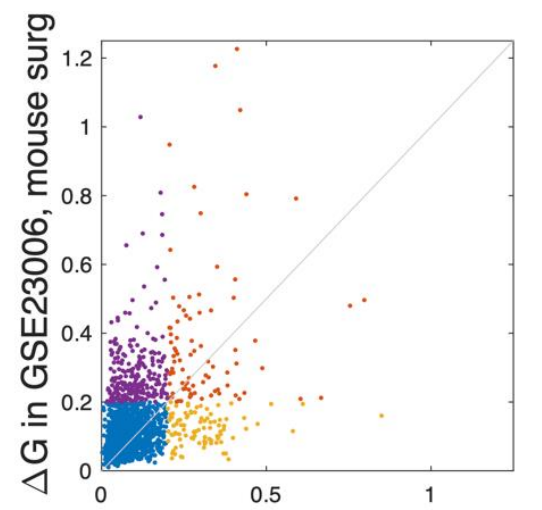

$\Delta \mathrm{G}$ in GSE8056, human burn

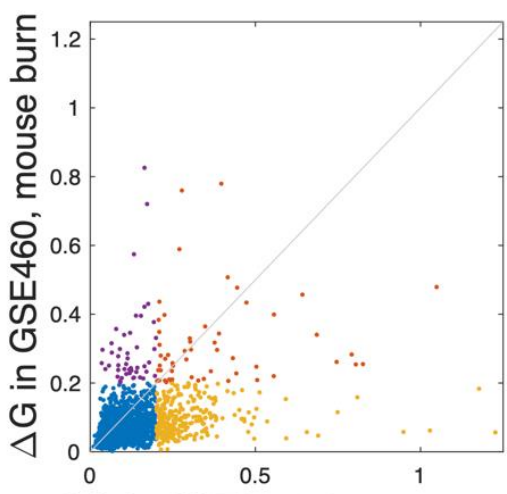

$\Delta G$ in GSE23006, mouse surg

122 Figure 2. Comparison of gene fold change $\Delta G$ between datasets for all intersecting genes. Each

123 point corresponds to one of 1622 genes shared by all 3 datasets. Different colors denote genes

124 with $\Delta G$ above (orange) or below (blue) 0.2 in both datasets as well as those with differing fold 125 changes across dataset (purple, yellow). 
127 Table 3. Spearman correlation coefficients between datasets calculated by different ranks.

\begin{tabular}{|l|l|l|l|}
\hline Datasets: & mouse surg vs & mouse surg vs & mouse burn vs \\
Rank type & mouse burn & human & \\
\hline Fold change $\Delta G$ & 0.42 & 0.36 & 0.27 \\
\hline Max upregulation & 0.39 & 0.34 & 0.22 \\
$\Delta G_{u}$ & & & \\
\hline Max downregulation & 0.22 & 0.29 & 0.15 \\
$\Delta G_{d}$ & & & \\
\hline
\end{tabular}

129 The distribution of $\Delta G$ is similar for all 3 datasets (Figure 1), however, the correlation between 130 datasets is weak (Table 3), i.e. if the gene has high/low $\Delta G$ in one wound, it does not necessarily 131 imply similar dynamics in another wound (Figure 2).

\section{Challenges in comparing gene expression dynamics between datasets}

134 Low correlation between gene expression fold change in different datasets may be explained by

135 an inconsistency in measurement time points. Figure 3a shows the dynamics of one gene (II1a)

136 with different values of $\Delta G$ in 3 datasets; the plots look very different. The mouse surgical wound

137 dataset contains eight timepoints over 10 days, while the mouse burn dataset contains only four

138 timepoints over 14 days. Some genes are upregulated in the early wound healing stages. The

139 mouse surgical wound, which contains measured timepoints between 2 and 72 hours, is able to 
141 measured timepoints in this time window. Thus, any potential transients would be missed. For 142 example, even if Illa would have upregulation in this period (dashed plots in Fig. 3b) in burn

143 wounds, similarly to mouse surgical wound, this cannot be seen in the burn wounds.

144 The same is observed for many other genes; in this work they are filtered out.

145

146

147
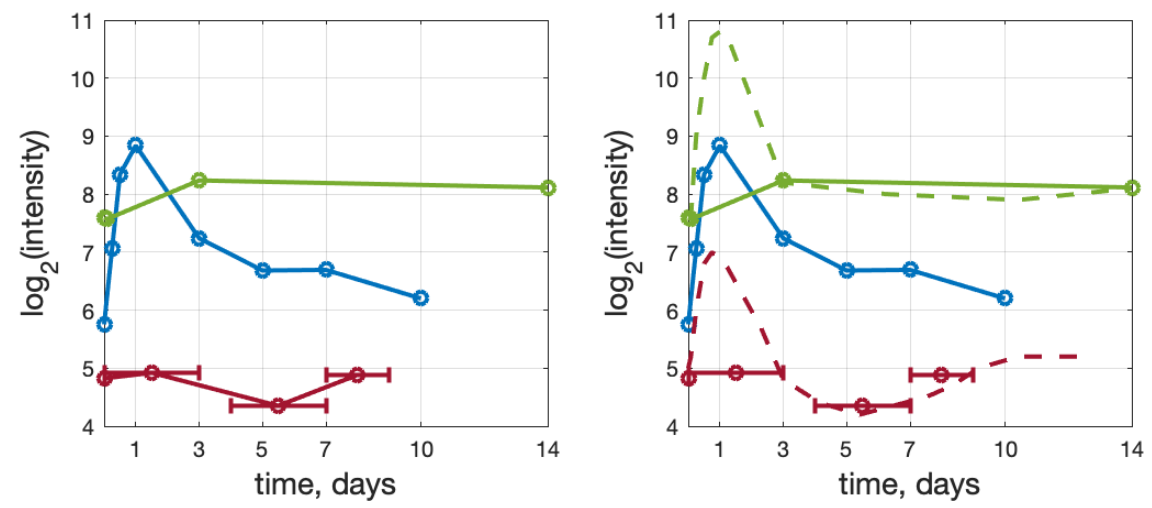

-

$\rightarrow$ mouse burn, $\Delta \mathrm{G}=0.09$

10r human burn, $\Delta \mathrm{G}=0.13$

- - mouse burn (interpolation)

- - human burn (interpolation)

Figure 3. (a) Plots of Il1a expression change vs time: comparison between wound datasets. (b)

The same gene dynamics along with possible gene expression plots (fake plots) corresponding to data but not captured due to lack of time points (dashed line).

\section{Commonly highly expressed genes}

We search the genes having high $\Delta G$ in each dataset. As described above, there are 1622

genes that appear in all three datasets after filtration. As there is no natural cutoff for $\Delta G$ values

(Figure 1) we select the first 300 genes with the highest $\Delta G$ from each dataset. The intersection of 3 subsets of 300 genes is 58 genes only. The plots of these 58 genes' dynamics in 3 wound datasets are presented in Figure 4.

157 mouse surgical data, where peak time refers to the window in which $\Delta G$ is maximized. Cluster1 
158 includes genes with peak time in mouse surgical wounds equal to $0-12 \mathrm{~h}$, Cluster2 - 24h, Cluster3

$159-72 \mathrm{~h}$, Cluster $4-120 \mathrm{~h}$ and Cluster $5>120 \mathrm{~h}$. Next the plots of the same genes in other datasets 160 were divided into the same cluster as GSE23006 mouse surgical, independently of their dynamics

161 in other datasets.

162 We generally observe similar dynamics for the genes in each cluster. In contrast the 163 clusters for each dataset exhibit different dynamics as can be seen by comparing images on the 164 same row.

165 Compared to the gene dynamics within a cluster, there is less similarity of dynamics 166 between wounds: the same cluster of genes demonstrates only roughly comparable dynamics 167 between the wounds (compare images within the same column). The genes of the first cluster 168 are downregulated from 0 to $72 \mathrm{~h}$ in all datasets and genes of the $3^{\text {rd }}$ cluster are mainly 169 upregulated at the same interval. However, if we compare the last cluster of genes, we see early 170 downregulation of genes in the surgical wound that is not mirrored in either of the burn wounds.

171 The unexpected outcome in this latter case may be due to the low-resolution time steps masking 172 the underlying dynamics.

173

174 

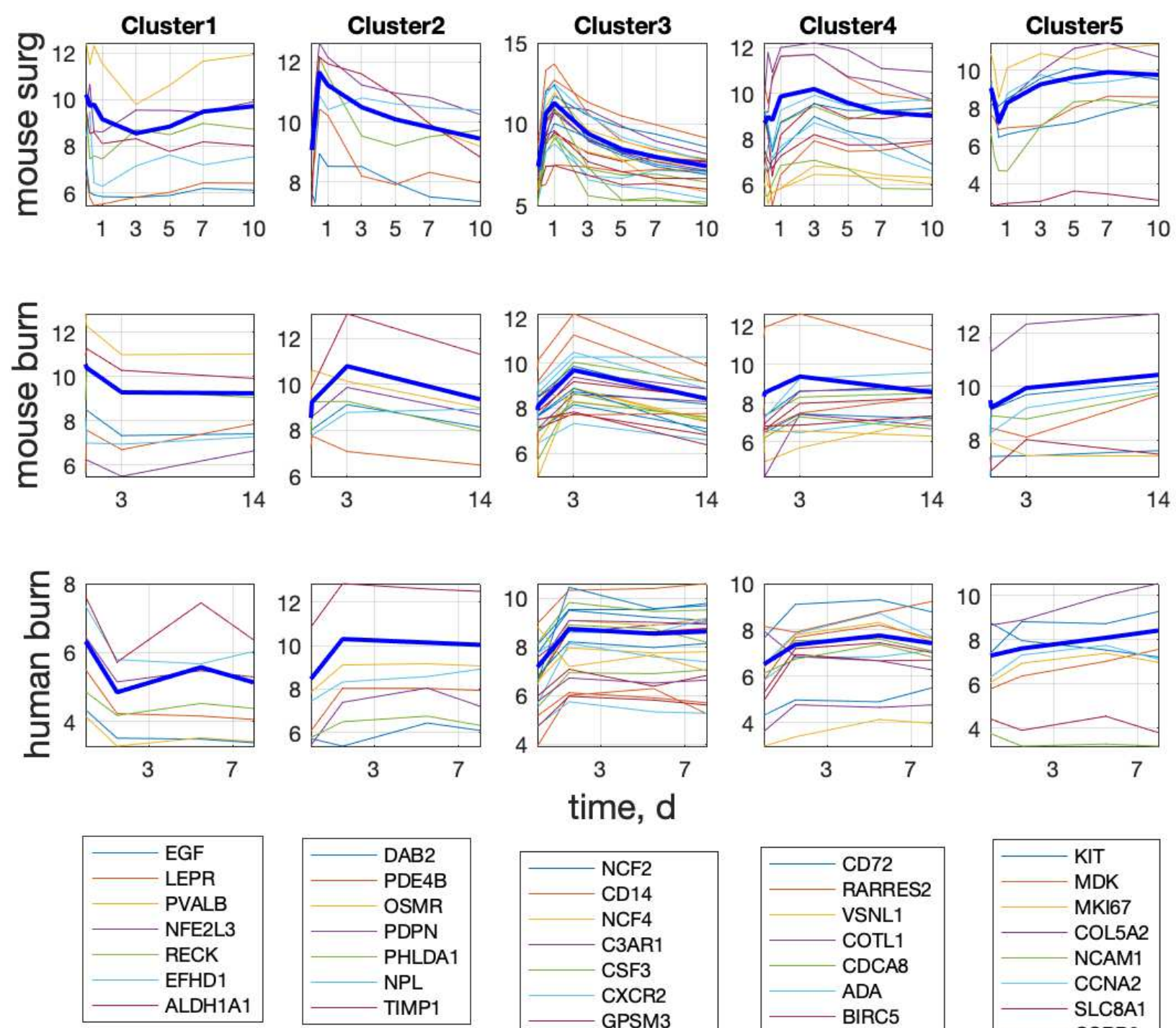

\begin{tabular}{|c|}
\hline NCF2 \\
CD14 \\
NCF4 \\
C3AR1 \\
CSF3 \\
CXCR2 \\
GPSM3 \\
ITGB2 \\
CD53 \\
CCR1 \\
HCK \\
HCLS1 \\
MARCKSL1 \\
PLSCR1 \\
PLAUR \\
CDC6 \\
HSD11B1 \\
SLC11A1 \\
SLC2A3 \\
ADAM8 \\
CREM \\
LILRB3
\end{tabular}

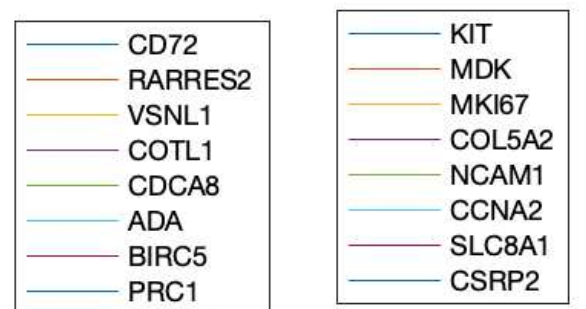

176 Figure 4. Gene expression dynamics of commonly highly expressed genes in the 3 datasets. Each

177 row represents the same dataset and each column represents the same cluster of genes listed 
178 in the legend under each column. Bold blue line in each plot corresponds to the mean value of

179 gene intensity within each cluster (calculated for each dataset separately). Vertical axis:

$180 \quad \log _{2}($ Intensity), horizontal axis - time in days.

181

182 To finalize comparison of gene expression between the wounds, we normalize the mean cluster

183 value by dividing by its initial value ( $t=0$, non-injured tissue):

$$
\text { Normalized mean cluster value }=\frac{J_{i}}{J_{0}}
$$

$$
J_{i}=\frac{1}{N} \sum_{k}^{N} g_{k}\left(t_{i}\right)
$$

where summation is over all genes within the cluster and $\mathrm{N}$ is number of genes in the cluster.

187 In Figure 5 normalized mean cluster value dynamics from the 3 datasets is collected.

188
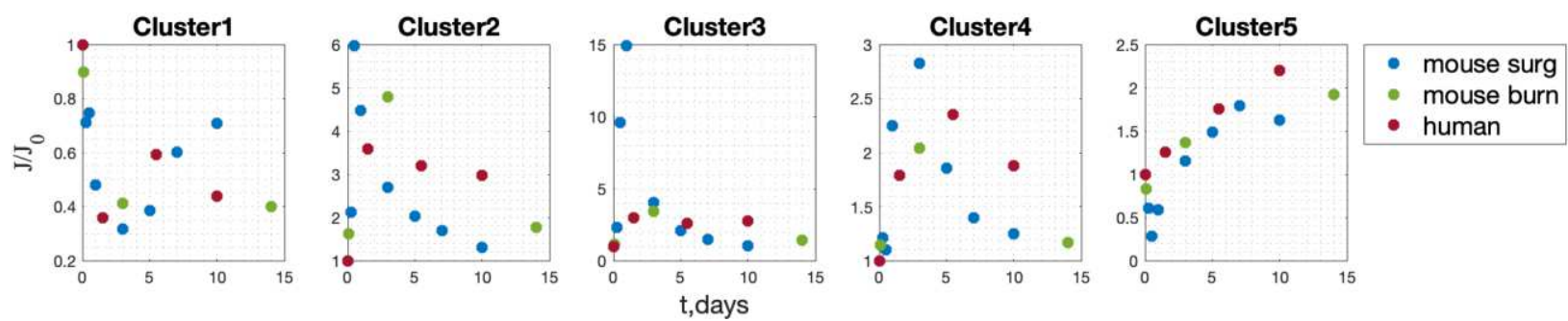

Figure 5. Normalized mean cluster value dynamics in three wound datasets. Clusters indicated

in Fig.4.

192 One can see that all five normalized mean cluster gene expression values have very similar

193 dynamics between wound datasets. Thus, we found the set of genes that could be used as 194 universal indicators of wound stage detection in different wounds.

\section{Discussion}


Wound healing, independently of wound origin, is traditionally divided into phases:

198 homeostasis, inflammation, proliferation, and remodeling [Canedo-Dorantes 2019]. Though skin

199 repair in mice does not perfectly mirror that of humans, prior experiments have shown that skin

200 healing is similar in humans and mice [Zomer 2017]. This work supports our assumption that

201 universal features of wound healing may be found even in different wound transcriptomic 202 datasets.

$203 \quad$ Wound transcriptomic datasets are not numerous. In fact, we have only few wound 204 experiments collected under different conditions [Raudys, 1991]. However, following [Sass 205 2017], we compared existing wound data, but focused on finding genes with similar dynamics. 206 We found 5 clusters of genes whose dynamics were similar in 3 available datasets. These are 207 strong candidates to universal biomarkers of known wound healing stages.

208 During our comparison analysis we found several methodological problems that hopefully 209 will be solved in the future.

210 Wound healing is a dynamical process. Experimental data collection, such as gene 211 expression intensity, should be done with appropriate frequency (sampling rate). In signal 212 processing the theorem of Nyquist-Shannon limits the frequency of discrete sampling to obtain 213 satisfactory information from a continuous signal [Dieter, 1999]. The sampling rate frequency 214 must be twice larger than the highest frequency of the signal. In many existing wound 215 transcriptomic datasets, the sampling rate doesn't satisfy this condition (see Figure 3). 216 Characteristic healing times depend on wound size, depth, experimental conditions, and overall 217 organism's health, etc. [Hess 2008; Canedo-Dorantes 2019; Hadian 2020]. Wound 
218 transcriptomics experiments are expansive and raise ethical questions, however, sampling rate

219 frequency must be taken into account when planning such experiments.

221 It is traditional in this field to assume high fold change in gene expression as high

222 significance of the gene. However low sample rate may lead to missing gene expression peak

223 (burn wounds in Fig 3) and misinterpretation of its significance. On the other hand, in the fold-

224 change values of genes there is no "bi-modal" distribution (highly changing and no-changing

225 dynamics) (Figure 1). Whether or not two-fold change or three-fold change expression is

226 considered significant is an arbitrary choice of the researcher. If high fold change is equivalent to

227 the "significance", then the significance of genes decreases gradually, there are no "significant"

228 and "non-significant" genes. Probably, the choice of the first $\mathrm{N}$ genes with the highest fold-

229 change in expression is more reasonable option to select the most significant genes.

230 The development of transcriptome technology is important for understanding wound

231 healing and for the improvement of intelligent methods of wound treatment. However, much

232 work is still to be done. For instance, transcriptomic data are usually not accompanied by

233 additional data, such as histology/morphology of the wound tissue. In fact, transcriptomic signals

234 are taken from many cells at once, and any information about the cell types in the wound at each

235 time would give much more insight into understanding of gene expression in wound healing.

236 In this work we performed wound transcriptomic dataset comparisons without any

237 reference to cells or biological processes and found a set of 58 genes that could serve as

238 indicators of wound healing stage. We used a filtering approach which allowed for comparisons

239 between species and was agnostic to experimental set up. The results were a set of 58 genes 
240 which may be used as a basis for future analysis. Potentially this set of biomarkers, or an

241 improved set pending further data collection and analysis, can be used in biomarkers toolkit for

242 gene-expression based wound stage detection.

\section{Methods}

245 In each dataset the intensity of gene expression is measured at multiple time points. In GSE23006

246 the intensities are represented as log2(intensity) while in GSE460 and GSE8056 datasets original

247 intensities are shown. To make datasets comparable, we return GSE23006 to initial intensity

248 before filtering by taking power of 2 of the numbers represented in the GSE23006 array.

\section{Orthologous genes between mouse and human}

250 To compare particular genes between human and mouse, we find orthologs - homologous genes

251 between species. All orthologous genes in mouse and human were matched by gene symbol to

252 their homologene ID in the Human and Mouse Homology Class report. (Source:

253 http://www.informatics.jax.org/homology.shtml).

254 The genes are considered for further analysis only if orthologs are found in all datasets under

255 consideration.

\section{Filtering Data of multiple genes in the same row (1)}

257 Although most probes in microarray transcriptomics exhibit a one-to-one mapping of probe-to-

258 transcript, this is not always the case. Similarities between the nucleotide sequences of different

259 genes can result in non-unique mappings, witnessed in the form "gene1//gene2//gene3" in the

260 datasets. To avoid faulty comparisons, we simply remove these rows from analysis.

\section{Filtering Data Based on consistency of replicates (2)}


262 In all considered datasets each of $\mathrm{n}$ genes is presented as 3 replicates in $\mathrm{m}$ time points. We first 263 denote the time points by $t_{j}$ such that $j=0, \ldots, m-1$, where the unwounded state is associated

264 with $j=0$. Let $G^{k}$ be an Rnxm matrix composed of $\mathrm{m}$ time series gene intensity measurements

265 for kth replicate of each of $n$ genes. Let the average gene intensity across the three replicates be 266 given by

$$
G=\frac{\sum_{k=1}^{3} G^{k}}{3} \in R^{n \times m}
$$

268 Where the division operator is applied component wise. Then the percent relative error for each 269 replicate is given by

$$
S^{k}=\frac{\left|G^{k}-G\right|}{G} * 100 \in R^{n \times m},
$$

271 with component wise operation in the division. Next we want to find the average relative error

272 for each time point across all genes and its standard deviation. This gives a sense of how much 273 each replicate deviates from the average across the replicates independent of the gene. Let the

274 matrix

$$
S=\left[S^{1} S^{2} S^{3}\right] \in R^{3 n \times 8}
$$

276 be a matrix composed of the matrices $S^{k}$. Then we take the average across the columns such

277 that we arrive at a row vector where each entry $i$ contains the average value across all elements

278 in column $i$ of matrix $S$. We denote this vector by $\vec{r}_{A V G} \in R^{1 \times 8}$ Similarly, we compute the standard 279 deviation across the columns of $S$ and denote this vector by $\vec{r}_{S T D} \in R^{1 \times 8}$. 
281 Now we compute the threshold for the maximum relative error, which will determine which data

282 is kept and which is discarded based on an acceptable value for relative error. The threshold for

283 each time point is chosen to be as follows

$$
\vec{r}_{\text {Thres }}=\vec{r}_{A V G}+4 \vec{r}_{S T D}
$$

286 where we take four standard deviations above the mean which is inclusive of $99.98 \%$ percent of 287 data assuming a normal distribution (Note that three standard deviations is inclusive of 99.72\%).

288 We find the maximum relative error across the three samples, where the new matrix $S_{\max }=$ $289 \max \left(S^{1}, S^{2}, S^{3}\right) \in R^{n \times m}$. The maximum is taken element wise across the three matrices, that is $290 S_{\max }(i, j)=\max \left(S^{1}(i, j), S^{2}(i, j), S^{3}(i, j)\right)$ and do an element-wise comparison across each row 291

$$
S_{\text {max,(i,:) }}>\vec{r}_{\text {Thres }}
$$

292 for $i=1: n$ and any time-series containing an extreme outlier in any of the replicates at any time

293 point is removed from the dataset and, hence, the row removed from matrix $G$ containing the

294 average intensity across the replicates. Note that we treat each time-point individually since 295 there may exist different degrees of variability through the different wound healing stages. We 296 denote the new matrix $\widehat{G}$, which contains a subset of the rows of $G$, after discarding rows with 297 high variability across replicates.

299 Filtration of repeated measurements of same gene in each dataset (3)

300 Some genes are mentioned in the dataset several times (several repetitions or several 301 transcripts). In addition, for some genes the repeated rows contain too different dynamics. To 302 leave "one gene - one row" we make filtering based on correlation between repeated gene rows. 
303 Denote the $\mathrm{i}^{\text {th }}$ row of the matrix $\hat{G}$ as $\vec{g}_{i} \in R^{1 \times m}$. It contains time point mean intensity

304 measurements of gene $i$ such that: $\vec{g}_{i}=\left[g_{i}\left(t_{0}\right), g_{i}\left(t_{1}\right), \cdots, g_{i}\left(t_{m}\right)\right]$. Suppose that there are $k$ 305 vectors corresponding to one and the same gene:

$306 \vec{g}_{i_{1}}, \ldots, \vec{g}_{i_{k}}$

307 First, we find Pearson correlation coefficients between each pair of repeated gene intensities:

$308 C_{n m}=\operatorname{corr}\left(\vec{g}_{n}, \vec{g}_{m}\right), n \neq m$, we obtain $k^{2}-k$ correlation coefficients. The gene is kept for 309 further analysis if at least two repetitions are highly correlated:

$$
\left(C_{n m}\right) \geq C
$$

311 In this work we use the threshold $C=0.9$. If the condition (*) is satisfied, we take one of highly

312 correlated gene intensity rows $\vec{g}_{n}, \vec{g}_{m}$ (we can take the mean between the intensities of two

313 highly correlated genes). If the condition $(*)$ is not satisfied, the gene is not included in further 314 analysis.

316 Several genes corresponding to the same homologene number (4)

317 For some genes it may happen that the same homologene number corresponds to two genes.

318 For example, homologene number corresponding to gene $\mathrm{X}$ in mouse corresponds to genes $\mathrm{X} 1$ 319 and $\mathrm{X} 2$ in human. In this case we check if there is high correlation between homologenes X1 and

$320 \times 2\left(\operatorname{corr}\left(\vec{g}_{X_{1}}, \vec{g}_{X_{2}}\right)>C\right)$ and take one of them. Otherwise, these homologenes are not included

321 in further analysis.

\section{Declarations}

\section{Ethics approval and consent to participate}

324 All procedures were performed in accordance with relevant guidelines. 


\section{Consent for publication}

326 Not applicable.

\section{Availability of data and materials}

328 Publicly available datasets used in this work: GSE23006, GSE460 and GSE8056 from Gene

329 Expression Omnibus database: https://www.ncbi.nlm.nih.gov/geo/

330 The filtered data created during the current study available from the corresponding author on

331 reasonable request.

\section{Competing interests}

333 The authors declare no competing interests.

\section{Funding}

335 Research was sponsored by the Office of Naval Research and the DARPA Biotechnologies Office 336 (DARPA/BTO) and was accomplished under Cooperative Agreement Number DC20AC00003. The

337 views and conclusions contained in this document are those of the authors and should not be

338 interpreted as representing the official policies, either expressed or implied, of the Office of Naval

339 Research and the DARPA Biotechnologies Office (DARPA/BTO) or the U.S. Government. The U.S.

340 Government is authorized to reproduce and distribute reprints for Government purposes

341 notwithstanding any copyright notation herein.

\section{Authors' contributions}

343 EM, KZ and MG developed the methods and drafted the manuscript. KZ performed data

344 analysis. MG guided the work. All authors discussed, read, revised, and approved the final

345 manuscript.

\section{Acknowledgements}


347 Not applicable.

\section{References}

349 1. L.Canedo-Dorantes, M. Canero-Ayala, Skin Acute Wound Healing: A Comprehensive Review // International Journal of Inflammation Volume 2019, Article ID 3706315.

2. M.H. Kim, W. Liu, D.L. Borjesson, F.R. Curry, L.S. Miller, A.L. Cheung, F.T. Liu, R.R. Isseroff, S.I. Simon, Dynamics of neutrophil infiltration during cutaneous wound healing and infection using fluorescence imaging, J Invest Dermatol 128(7) (2008) 1812-20.

3. P. Krzyszczyk, R. Schloss, A. Palmer, F. Berthiaume, The Role of Macrophages in Acute and Chronic Wound Healing and Interventions to Promote Pro-wound Healing Phenotypes, Front Physiol 9 (2018) 419.

4. P. Bainbridge, Wound healing and the role of fibroblasts, J Wound Care 22(8) (2013) 4078, 410-12.

5. I. Pastar, O. Stojadinovic, N.C. Yin, H. Ramirez, A.G. Nusbaum, A. Sawaya, S.B. Patel, L. Khalid, R.R. Isseroff, M. Tomic-Canic, Epithelialization in Wound Healing: A Comprehensive Review, Adv Wound Care (New Rochelle) 3(7) (2014) 445-464.

6. H.N. Wilkinson, M.J. Hardman, Wound healing: cellular mechanisms and pathological outcomes, Open Biol 10(9) (2020) 200223.

7. J.O. Brant, M.C. Lopez, H.V. Baker, W.B. Barbazuk, M. Maden, A Comparative Analysis of Gene Expression Profiles during Skin Regeneration in Mus and Acomys, PLoS One 10(11) (2015) e0142931. 
healing, J Transl Med 5 (2007) 11.

370 9. Blumenberg M. Introductory chapter: transcriptome analysis. In: Transcriptome Analysis. Blumenberg M (Ed.). IntechOpen, London, UK, 1-5 (2019).

10. C. Tachibana, Transcriptomics today: Microarrays, RNA-seq, and more. // Science 2015: Vol. 349, Issue 6247, pp. 544-546.

11. L.Chen, Z.H.Arbieva, S.Guo, P.T. Marucha, T.A. Mustoe, L.A. DiPietro, Positional differences in the wound transcriptome of skin and oral mucosa // BMC Genomics 2010, 11:471

12. Greco JA 3rd, Pollins AC, Boone BE, Levy SE et al. A microarray analysis of temporal gene expression profiles in thermally injured human skin. Burns 2010 Mar;36(2):192-204.

13. P.A. Sass, M. Dabrowski, A. Charzynska, P. Sachadyn, Transcriptomic responses to wounding: meta-analysis of gene expression microarray data. // BMC Genomics, 2017, V 18: 850.

14. J. Xue, S.V. Schmidt etc.,Transcriptome-Based Network Analysis Reveals a Spectrum Model of Human Macrophage Activation // Immunity. 2014 Feb 20; 40(2): 274-288.

15. Y.Du, P.Ren. Q.Wang, S.-K. Jiang, M.Zhang, J.-Y. Li, L.-L. Wang, D.-W. Guan, , Cannabinoid 2 receptor attenuates inflammation during skin wound healing by inhibiting M1 macrophages rather than activating M2 macrophages // Journal of Inflammation (2018) $15: 25$

16. S. Raychaudhuri, J.M. Stuart, R.B. Altman, Principal component analysis to summarize microarray experiments: application to sporulation time series. // Pac Symp Biocomput, $2000,455-66$. 
17. A.L. Tarca, R. Romero, S. Draghici, Analysis of microarray experiments of gene expression profiling. // Am J Obstet Gynecol. 2006, 195(2): 373-388.

18. S.J.Raudys, A.K.Jain, Small sample size effects in statistical pattern recognition: recommendations for practitioners. // IEEE Transactions on pattern analysis and machine intelligence, V 13 N 3 1991:252-264.

19. L.H. Dieter, "The Origins of the Sampling Theorem". 1999, IEEE Communications Magazine. 37 (4): 106-108.

20. Hess CT. Clinical Guide to Skin and Wound Care. 6th ed. Philadelphia, PA: Lippincott Williams \& Wilkins; 2008.

400

21. Y. Hadian, D. Fregoso, C. Nguyen, M.D. Bagood, S.E. Dahle, M.G. Gareau, R.R. Isseroff, Microbiome-skin-brain axis: A novel paradigm for cutaneous wounds, Wound Repair Regen 28(3) (2020) 282-292.

22. JL Martin, L Koodie, AG Krishnan, R Charboneau, RA Barke, S Roy, Chronic Morphine Administration Delays Wound Healing by Inhibiting Immune Cell Recruitment to the Wound Site// The American Journal of Pathology 2010

23. L. Chen, S. Nagaraja, J. Zhou, Y. Zhao, D. Fine, A.Y. Mitrophanov, J. Reifman, L.A. DiPietro, Wound healing in Mac-1 deficient mice, Wound Repair Regen 25(3) (2017) 366-376.

24. Evans TG. Considerations for the use of transcriptomics in identifying the 'genes that matter' for environmental adaptation. J Exp Biol. 2015 Jun;218(Pt 12):1925-35. doi: 10.1242/jeb.114306. PMID: 26085669.

25. Feder ME, Walser JC. The biological limitations of transcriptomics in elucidating stress and stress responses. J Evol Biol. 2005 Jul;18(4):901-10. doi: 10.1111/j.1420- 
414 26. Dalman, M.R., Deeter, A., Nimishakavi, G. et al. Fold change and p-value cutoffs 415 significantly alter microarray interpretations. BMC Bioinformatics 13, S11 (2012). $416 \quad$ https://doi.org/10.1186/1471-2105-13-S2-S11

417 27. McCarthy, Davis J, and Gordon K Smyth. "Testing significance relative to a fold-change 418 threshold is a TREAT." Bioinformatics (Oxford, England) vol. 25,6 (2009): 765-71. 419 doi:10.1093/bioinformatics/btp053

420 28. Zomer HD, Trentin AG. Skin wound healing in humans and mice: Challenges in 421 translational research. J Dermatol Sci. 2018 Apr;90(1):3-12. doi: 422 10.1016/j.jdermsci.2017.12.009. Epub 2017 Dec 26. PMID: 29289417. 